Research Paper

\title{
Visceral Adipose Tissue Modulates Radiosensitivity in Oesophageal Adenocarcinoma
}

\author{
Ann Marie Mongan ${ }^{1}$, Niamh Lynam-Lennon ${ }^{1}$, Suzanne L Doyle ${ }^{2}$, Rory Casey ${ }^{1}$, Eibhlin Carr ${ }^{3}$, Aoife

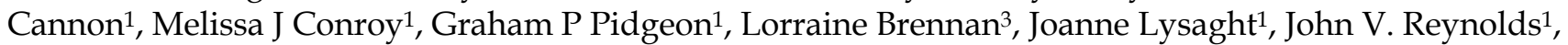 \\ Jacintha O'Sullivan ${ }^{1}$ \\ 1. Trinity Translational Medicine Institute, Department of Surgery, Trinity College Dublin, St. James's Hospital, Dublin 8, Ireland \\ 2. School of Biological Sciences, Dublin Institute of Technology, Dublin, Ireland. \\ 3. School of Agriculture \& Food Science, Science Centre-South, Belfield, Dublin 4, Ireland \\ $\triangle$ Corresponding author: Prof. Jacintha O'Sullivan, Professor in Translational Oncology; Telephone: +353 18962149; Email: osullij4@tcd.ie; Address: Trinity \\ Translational Medicine Institute, Department of Surgery, Trinity College Dublin, St. James's Hospital, Dublin 8, Ireland. \\ (c) Ivyspring International Publisher. This is an open access article distributed under the terms of the Creative Commons Attribution (CC BY-NC) license \\ (https://creativecommons.org/licenses/by-nc/4.0/). See http://ivyspring.com/terms for full terms and conditions.
}

Received: 2018.08.16; Accepted: 2018.10.13; Published: 2019.04.05

\begin{abstract}
Oesophageal adenocarcinoma $(\mathrm{OAC})$ is an exemplar model of obesity-associated cancer. Response to neoadjuvant chemoradiotherapy (NA CRT) is a clinical challenge. We examined if visceral adipose tissue and obesity status alter radiosensitivity in OAC.

The radioresistant (OE33R) and radioresponsive (OE33P) OAC isogenic model was cultured with adipose tissue conditioned media from three patient cohorts: non-cancer patients, surgery only OAC patients and NA CRT OAC patients. Cell survival was characterised by clonogenic assay, metabolomic profiling by nuclear magnetic resonance spectroscopy and adipokine receptor gene expression by qPCR. A retrospective in vivo study compared tumour response to NA CRT in normal weight $(n=53)$ versus overweight/obese patients $(n=148)$.

Adipose conditioned media (ACM) from all patient cohorts significantly increased radiosensitivity in radioresistant OE33R cells. ACM from the NA CRT OAC cohort increased radiosensitivity in OE33P cells. Metabolomic profiling demonstrated separation of the non-cancer and surgery only OAC cohorts and between the non-cancer and NA CRT OAC cohorts. Gene expression profiling of OE33P versus OE33R cells demonstrated differential expression of the adiponectin receptor-1 (AR1), adiponectin receptor-2 (AR2), leptin receptor (LepR) and neuropilin receptor-1 (NRPI) genes. In vivo overweight/obese OAC patients achieved an enhanced tumour response following NA CRT compared to normal weight patients. This study demonstrates that visceral adipose tissue modulates the cellular response to radiation in OAC.
\end{abstract}

Key words: oesophageal cancer, obesity, radiotherapy, visceral adipose tissue

\section{Introduction}

Oesophageal adenocarcinoma (OAC) is an aggressive disease with five-year survival rates of approximately $20 \%$, and $40 \%$ for patients who are treated with curative intent (1). For locally advanced disease, neoadjuvant chemoradiation (NA CRT) is increasingly the standard of care (2-4). The CROSS trial demonstrated that NA CRT followed by surgery was associated with a greater than two-fold increase in median overall survival compared with surgery alone (2,3). A meta-analysis including CROSS and eleven randomised controlled trials (RCTs) of multimodal therapy compared with surgery alone concluded that NA CRT was associated with a significant improvement in overall survival (4). Tumour response to NA CRT predicts survival, but resistance to therapy remains a significant clinical problem with only $14-45 \%$ of patients achieving a complete pathological response (pCR) (2-4).

OAC is an examplar model of an obesityassociated cancer, and obesity may underlie the marked increase in the incidence of OAC (1,5-7). Although the epidemiologic association between obesity and adenocarcinoma is well described, the impact of obesity on tumour biology, response to therapy, and outcomes, is unclear. Several studies have shown that obesity is associated with increased 
risk of cancer recurrence and death, (8-10), while others have reported no difference in survival between obese and non-obese patients (11-14). Importantly, obesity status may play differential roles in tumour development during treatment and following surgery.

The relationship between visceral adipose tissue (VAT) and radioresponse has not been investigated. The radioresistant phenotype is correlated with features such as altered DNA repair (15), cell cycle checkpoint operation (15), telomere biology (16), reactive oxygen species (ROS) biology (17), and induction of apoptosis (15), all of which are hypothesized to be affected by visceral adiposity. Obesity is associated with a state of low-grade chronic inflammation that causes systemic oxidative stress $(18,19)$, a mechanism by which ionizing radiation can damage cells (17). Obesity is associated with alterations in DNA repair mechanisms (20), and radioresistance of OAC is associated with DNA damage repair efficiency (21). Other putative mechanisms linking obesity and aggressive tumour behaviour include adipokine production, the insulin-like growth factor axis and sex steroids (22). Alterations in tumour biology are mediated not by adipocytes, but predominantly by secreted factors from the adipose tissue stromal vascular fraction (23). We have shown that visceral adipose conditioned media (ACM) in OAC patients is a rich source of adiponectin, leptin and VEGF (24), and metabolomic profiling has demonstrated an altered profile in ACM from viscerally obese compared to non-obese OAC patients (25). This current study assesses the interaction between obesity and radioresponse in vitro and in vivo in OAC.

\section{Methods}

\section{OAC tumour and adipose tissue biobank}

Histologically proven OAC patients undergoing surgical resection, and elective non-oncological gastrointestinal surgeries were recruited to the study with patient consent. Full ethical approval was granted by the Adelaide and Meath Hospital (incorporating the National Children's Hospital) $(\mathrm{AMNCH})$ research ethics committee. Body weight, height, visceral fat area (VFA) and central waist circumference (WC) were measured. Body mass index (BMI) was calculated as weight $(\mathrm{kg}) /$ height $^{2}\left(\mathrm{~m}^{2}\right)$. WC was measured to the nearest $0.1 \mathrm{~cm}$ using a tape measure midway between the uppermost border of the iliac crest and the lower border of the costal margin in the midaxillary line after respiratory exhalation while the patient was in a standing position (26). VFA was calculated from a pre-operative diagnostic computed tomography (CT) scan by an experienced radiologist, using a validated technique $(27,28)$. A VFA in excess of $163.8 \mathrm{~cm}^{2}$ (men) and $80.1 \mathrm{~cm}^{2}$ (women) was classified as obese, or WC in excess of $94 \mathrm{~cm}$ (men) and $80 \mathrm{~cm}$ (women) was used where VFA wasn't available (26). Standard operating procedures for the collection, storage and analysis of specimens were used (29).

\section{Visceral adipose tissue processing and cell co-culture with visceral ACM}

Omental adipose tissues were excised at the beginning of the surgical resections; adipose tissue was minced, washed with sterile PBS, and cultured in M199 media (Gibco) (5 g adipose tissue in $10 \mathrm{~mL}$ media) for 72 hours. The adipose tissue culture was filtered to remove adipose tissue fragments and the supernatant $(\mathrm{ACM})$ stored at $-80^{\circ} \mathrm{C}$. The OE33P and OE33R isogenic model of radioresistance were cultured as previously described (21) and treated with M199 control media or ACM at $37^{\circ} \mathrm{C}$ in $95 \%$ humidified air and $5 \% \mathrm{CO}_{2}$ for 24 hours.

\section{Clonogenic assay to assess radiosensitivity}

OE33P and OE33R cells were co-cultured for 24 hours with M199 control media or ACM, cells were irradiated with 0 or 2 Gy X-ray radiation using a Gulmay Medical X-ray generator, (RS225) (Gulmay Medical), at a dose rate of 3.25 Gray (Gy) per minute. Cells were cultured for 7-14 days to allow surviving colonies to reach maximum density without merging of colonies. Cells were stained with crystal violet dye for 30 minutes, dye removed and colonies consisting of 50 cells or more counted. Plating efficiencies (PE), which are the fraction of colonies from untreated cells, were calculated using the formula: $\mathrm{PE}=$ No. colonies / No. cells seeded. The surviving fraction (SF), which is the number of colonies produced after treatment, expressed in terms of $\mathrm{PE}$, was calculated using the formula: $\mathrm{SF}=$ No. colonies $/($ No. cells seeded $\times \mathrm{PE})$.

\section{Metabolomic profiling}

A volume of $250 \mu \mathrm{L}$ deuterium and $10 \mu \mathrm{L}$ sodium trimethyl $\left[2,2,3,3-{ }^{2} \mathrm{H}_{4}\right]$ propionate (TSP) $(0.005$ $\mathrm{g} / \mathrm{mL}$ ) were added to each $300 \mu \mathrm{L}$ ACM sample. Spectra were acquired on a $600-\mathrm{MHz}$ Varian nuclear magnetic resonance (NMR) spectrometer (Varian Limited, Oxford, United Kingdom) by using the first increment of a NOESY (Nuclear overhauser effect spectroscopy) pulse (sequence at 251C). ${ }^{1} \mathrm{H}$ NMR ACM spectra were processed with Chenomx software (version 6; Chenomx Edmonton, Canada) and were phase and baseline corrected. Spectra were integrated into bins consisting of spectral regions of $0.001 \mathrm{ppm}$. The water region was excluded, and data normalized to the total area of the spectral integral. Discriminating metabolites were identified by using libraries of 
pure metabolites developed in-house and the Chenomx database library. Acquisition of samples resulted in spectra containing a number of peaks, representing proton resonance in ${ }^{1} \mathrm{H}$ NMR.

\section{Adipokine receptor gene expression following co-culture with ACM}

Following co-culture of OE33P and OE33R cells with ACM, RNA was isolated using TriReagent and reverse transcribed as previously described (25). Adipokine gene expression (Adiponectin Receptor 1, Adiponectin Receptor 2, Leptin Receptor, Neuropilin1 ), was measured by qPCR using a TaqMan ${ }^{\circledR}$ assay kit (Applied Biosystems), and 18S was used as an endogenous control for data normalisation. Analysis was performed using SDS 2.3 and SDS RQ 1.2 relative quantification software (Applied Biosystems). One sample was set as the calibrator for analysis. Gene expression was expressed as fold difference in expression relative to control.

\section{Retrospective analysis of patient associations between obesity and radiation sensitivity}

A retrospective analysis of patients with resectable oesophageal or oesophagogastric junction adenocarcinoma treated with NA CRT (neoadjuvant chemoradiotherapy) at St. James's Hospital was performed. Clinical, pathological and survival data were retrieved from our cancer database. All resected tumours were staged in accordance with the American Joint Committee on Cancer (AJCC) (30). The extent of residual carcinoma in the surgical specimen was assigned a tumour regression grade (31). Tumour regression grade (TRG) 1-3 was considered to represent a good response to therapy, TRG $4-5$ a poor response to therapy. Response to therapy was also classified accordinng to the modified Mandard three-point TRG that distinguishes complete, partial, and minimal or non-responders (32).

\section{Statistical Analysis}

Statistical analysis was performed using Graphpad Prism 5 software and SPSS version 18 for Windows software. Unless otherwise stated, data were expressed as mean \pm standard error of the mean (SEM). Clinical data was expressed as the median. Unpaired $t$-tests and the Mann-Whitney $U$ test were used to examine statistical significance between unpaired groups of parametric and non-parametric data respectively. Categorical variables were analysed by Chi-square and Fisher's exact tests. One-way analysis of variance (ANOVA) and Tukey multiple comparisons post-hoc test were performed where the number of groups was three or more. RR refers to relative risk, $95 \% \mathrm{CI}$ to $95 \%$ confidence interval.
Survival analysis was performed using Kaplan-Meier curves and log-rank test and calculated from date of diagnosis to the first event (recurrence, progression, death or end of the follow-up period).

For metabolomic data analyses, multivariate metabolite data analyses were performed with Simca$\mathrm{P}+$ software (version 11.0; Umetrics, Umea ${ }^{\circ}$, Sweden). The ${ }^{1} \mathrm{H}$ NMR spectra were analysed using two pattern recognition methods; principal component analysis (PCA) (unsupervised) sets to explore any overall trends in the data; and partial least square discriminant analysis (PLS-DA). The variable importance in the projection (VIP) value of each variable in the model was calculated to indicate its contribution to the classification of samples. Variables with a VIP value $>1.5$ were considered important in discriminating between groups. The quality of all models was judged by the goodness-of-fit parameter $\left(\mathrm{R}^{2}\right)$ and the predictive ability parameter $\left(\mathrm{Q}^{2}\right)$, which is calculated by an internal cross-validation of the data and the predictability calculated on a leave-out basis. For all analysis, $p \leq 0.05$ was considered to be statistically significant.

\section{Results}

\section{Visceral ACM increases sensitivity to radiation in OE33P and OE33R cells}

Clonogenic assay was used to assess sensitivity to 2 Gy X-ray radiation in OE33P and OE33R cells following incubation with M199 control media or ACM from non-cancer patients (non-cancer cohort, $n=5$ ) (Figure 1A, B), ACM from OAC patients who had not undergone NA CRT prior to surgery (surgery only OAC cohort, $n=14$ ) (Figure 1C, D), and ACM from OAC patients who received NA CRT therapy prior to surgery (NA CRT OAC cohort, $n=10$ ) (Figure 1E, F). Anthropometric data for patient ACM used in clonogenic assay is summarised in supplementary table 1. OE33P cells incubated with ACM from the non-cancer and surgery only OAC cohort did not demonstrate any significant change in sensitivity to 2 Gy X-ray radiation, when compared to cells incubated with M199 control media (Figure 1A and C). OE33P cells treated with ACM from the NA CRT OAC cohort demonstrated a statistically significant increase in radiosensitivity to $2 \mathrm{~Gy}$, when compared to cells treated with M199 control media ( $p=0.009$, Figure 1E). Interestingly, OE33R cells were significantly more radiosensitive following co-culture with ACM from all three patient groups, the non-cancer cohort $(p=0.03$, Figure $1 \mathrm{~B})$, the surgery only OAC cohort $(p=0.002$, Figure 1D) and the NA CRT OAC cohort $(p<0.0001$, Figure 1F), compared to cells treated with M199 control media. Radiosensitivity was significantly increased in OE33R cells treated with ACM from 
NA CRT OAC patients compared to cells treated with $\mathrm{ACM}$ from the radiation naïve OAC patient cohort $(p=0.003$, Figure $1 G)$. The ACM used in this study was generated from obese and non-obese OAC patients, but no differences in survival fractions of OE33P and OE33R cells were observed when ACM from non-obese compared to obese patients was analysed (data not shown), indicating that it is the visceral adipose tissue itself which can increase radiosensitivity, particularly in cells that are radioresistant.

\section{Metabolomic profiling of ACM from three patient cohorts}

To investigate if the metabolites secreted in ACM differ between the three patient cohorts, we performed metabolomic profiling of ACM using nuclear magnetic resonance spectroscopy (NMR). Anthropometric data for patient ACM used in metabolomic screening is summarised in supplementary table 2 . A representtative PCA plot showing separation on the basis of the three patient cohorts (non-cancer, surgery only OAC, and NA CRT OAC patients) is depicted in Figure 2A $\left(\mathrm{R}^{2}=0.736\right)$. A robust PLS-DA model (Figure $2 \mathrm{~B}$ ) was built to further explore the differences $\left(\mathrm{R}^{2}=0.453, \mathrm{Q}^{2}=\right.$ 0.235). To aid comparison and identification or regions of difference a pairwise comparison was performed. The most influential peaks in the models were identified and a metabolite assigned to each peak. Semi-quantitative concentrations were compared between groups for the most influential metabolites.

A PCA plot of ${ }^{1} \mathrm{H}$ NMR ACM data for noncancer patients versus surgery only OAC patients showed separation of the two cohorts, $\left(R^{2}=0.699\right.$, Figure 2C). A PLS-DA model was built to compare ACM profiles from the two cohorts $\left(\mathrm{R}^{2}=0.479, \mathrm{Q}^{2}=\right.$ 0.304 , Figure 2D). Glucose was significantly increased $(p=0.019)$, whilst threonine $(p=0.014)$ lysine, $(p=0.017)$, and valine $(p=0.029)$ were all significantly decreased in ACM from non-cancer patients, when compared to surgery only OAC patients (Table 1A).

PCA and PLS-DA plots revealed separation between ACM from non-cancer and NA CRT OAC patients (Figure 2E, $\mathrm{R}^{2}=0.0474, \mathrm{Q}^{2}=0.318$ ). $\mathrm{ACM}$ from non-cancer patients demonstrated significantly increased glucose $(p=0.012)$ but significantly decreased threonine $(p=0.005)$ lysine, $(p=0.004)$, valine $(p=0.007)$, isoleucine $(p=0.039)$ and glycine $(p=0.05)$ when compared to NA CRT OAC patients (Table 1B).

\section{Adipokine receptor expression differs between OE33P and OE33R at baseline and following culture in visceral ACM}

Gene expression of four adipokine receptors representing abundant adipokines in non-obese and obese VAT was assessed in OE33P and OE33R cell

lines both at baseline and following co-culture with $\mathrm{ACM}$; adiponectin receptor 1 (AR1); adiponectin receptor 2 (AR2); leptin receptor (LepR); and the VEGF receptor neuropilin-1 (NRP1). Anthropometric data for patient ACM used in these analyses is shown in supplementary table 3.

(a)

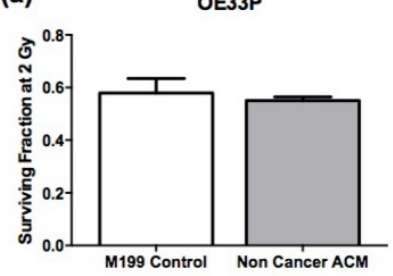

(c)

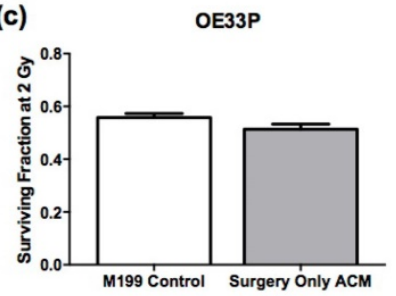

(e)

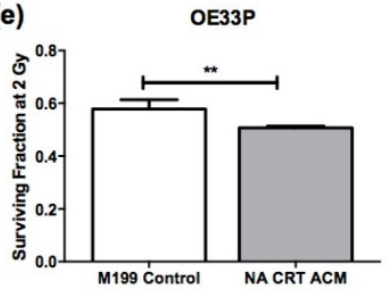

(g)

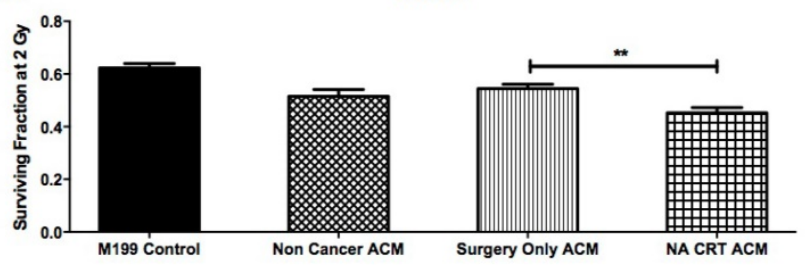

Figure 1. ACM increases radiosensitivity in OE33P and OE33R. OAC cells were treated with either M199 control media or ACM from non-cancer patients $(n=5), A C M$ from surgery only OAC patients (OAC patients who had not undergone NA CRT) $(n=14)$, or $A C M$ from OAC patients who had previously undergone NA CRT $(n=10)$ for $24 \mathrm{hrs}$, and sensitivity to 2 Gy X-ray radiation was assessed. (A) Co-culture of OE33P cells with ACM from non-cancer patients did not significantly alter radiosensitivity. (B) Co-culture of OE33R cells with ACM from non-cancer patients significantly increased radiosensitivity, when compared to $M 199$ control media $(p=0.03)$. (C) OE33P cells treated with ACM from surgery only OAC patients did not demonstrate significantly altered radiosensitivity. (D) OE33R cells treated with ACM from surgery only OAC patients demonstrated significantly increased sensitivity to 2 Gy, when compared to M199 control media $(P=0.002)$. (E) OE33P cells treated with ACM from NA CRT OAC patients demonstrated significantly increased sensitivity to $2 \mathrm{~Gy}$, when compared to $M 199$ control media $(p=0.009)$. (F) OE33R cells treated with ACM from NA CRT OAC patients demonstrated significantly increased sensitivity to $2 \mathrm{~Gy}(\mathrm{p}<0.0001)$, when compared to M199 control media. (G) OE33R cells treated with ACM from NA CRT OAC patients showed significantly increased sensitivity to $2 \mathrm{~Gy}$, when compared to cells treated with ACM from surgery only OAC patients $(p=0.003)$. Data are expressed as mean \pm SEM. Statistical analysis was performed using unpaired student's $t$ test. Statistical analysis was performed using one-way ANOVA with Tukey post-hoc correction. ${ }^{*} \mathrm{p}<0.05$, ${ }^{* *} \mathrm{p}<0.001$, ${ }^{* * *} \mathrm{p}<0.0001$ 
Table 1A. Metabolites discriminating non-cancer patients versus surgery only OAC patients, and non-cancer patients versus NA CRT OAC patients. A. VIPs generated from the PLS-DA of non-cancer patients versus surgery only OAC patients

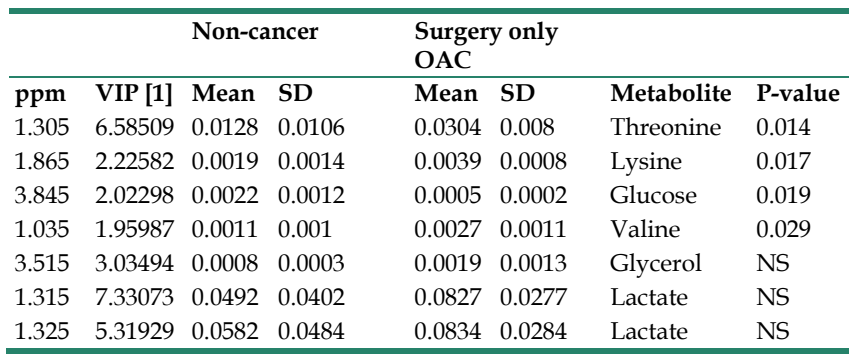

Glucose was significantly increased $(p=0.019)$, whilst threonine $(p=0.014)$ lysine, $(p=0.017)$, and valine $(p=0.029)$ were all significantly decreased in ACM from non-cancer patients, when compared to surgery only OAC patients.

VIP scores are indicated. For each metabolite, only peak is presented here. VIP= Variable in the Projection; PLS-DA= Partial least square discriminant analysis; PPM=Parts per million; NS=Non-significant

Table 1B. VIPs generated from the PLS-DA of non-cancer patients versus NA CRT OAC patients

\begin{tabular}{|c|c|c|c|c|c|c|c|}
\hline \multirow[b]{2}{*}{ ppm } & \multirow[b]{2}{*}{ VIP [2] } & \multicolumn{2}{|c|}{ Non-cancer } & \multicolumn{2}{|c|}{ NA CRT OAC } & \multirow[b]{2}{*}{ Metabolite } & \multirow[b]{2}{*}{ P-value } \\
\hline & & Mean & SD & Mean & SD & & \\
\hline 1.295 & 5.67466 & 0.0005 & 0.006 & & 0.0042 & Threonine & 0.005 \\
\hline 1.885 & 2.18606 & 0.0013 & 0.0005 & 0.0029 & 0.001 & Lys & 0.004 \\
\hline 3.745 & 2.09995 & 0.0025 & 0.001 & 0.0011 & 0.0002 & Glucose & 0.012 \\
\hline 1.035 & 2.26945 & 0.0011 & 0.001 & 0.0029 & 0.0008 & Valine & 0.007 \\
\hline 3.535 & 5.24455 & 0.0083 & 0.0076 & 0.0205 & 0.0111 & Gly & 0.05 \\
\hline 1.225 & 2.15509 & 0.0011 & 0.0017 & 0.003 & 0.0011 & Isole & 0.039 \\
\hline 1.445 & 3.68593 & 0.0085 & 0.0072 & 0.0023 & 0.0006 & Alanine & NS \\
\hline \multicolumn{8}{|c|}{$\begin{array}{l}\text { ACM from non-cancer patients demonstrated significantly increased glucose } \\
(p=0.012) \text { but significantly decreased threonine }(p=0.005) \text { lysine, }(p=0.004) \text {, valine } \\
(p=0.007) \text {, isoleucine }(p=0.039) \text { and glycine }(p=0.05) \text { when compared to NA CRT } \\
\text { OAC patients. } P \text {-values are based on unpaired t-test. }\end{array}$} \\
\hline \multicolumn{8}{|c|}{$\begin{array}{l}\text { VIP scores are indicated. For each metabolite, only peak is presented here. VIP= } \\
\text { Variable in the Projection; PLS-DA= Partial least square discriminant analysis; NA } \\
\text { CRT=Neoadjuvant chemoradiotherapy; PPM=Parts per million; NS=Non } \\
\text { significant }\end{array}$} \\
\hline
\end{tabular}

Expression of AR1 was significantly higher in OE33P cells compared to OE33R cells at baseline $(p=0.008)$ (Figure 3A). There was a two-fold downregulation in AR1 expression in OE33P cells following 24 hours of ACM treatment compared to cells treated with control media $(p=0.019)$. ACM treatment did not significantly alter AR1 expression in OE33R cells (Figure 3B). Expression of AR2 was significantly higher in OE33P cells compared to OE33R cells at baseline $(p=0.015)$ (Figure 3C). AR2 expression following ACM treatment was not altered in OE33P or OE33R (Figure 3D).

Interestingly, levels of LepR were significantly lower in OE33P cells compared to OE33R cells at baseline $(p=0.033)$ (Figure 3E). LepR expression was five-fold lower in OE33P compared to OE33R cells treated with ACM $(p=0.0004)$, (Figure 3F). Levels of NRP1 were also significantly lower in OE33P cells compared to OE33R cells at baseline $(p=0.038)$ (Figure $3 G)$. ACM induced a 13-fold upregulation in NRP1 expression in OE33P cells ( $p=0.047)$, but did not alter NRP1 expression in OE33R cells (Figure 3H).

\section{Overweight and obese OAC patients demonstrate enhanced radiosensitivity compared to normal weight patients}

Between 1990 and 2012, 201 consecutive OAC patients completed neoadjuvant chemoradiotherapy (NA CRT) as part of their curative therapy, and had a surgical resection. Clinical and pathological data for this cohort is summarised in supplementary table 4 .

Overweight/obese patients were more likely to have a lower pathological $\mathrm{T}$ stage at surgery following NA CRT, when compared to normal weight patients, (Relative Risk (RR) 1.495, 95\% Confidence Interval (CI) 1.094-1.938, $p=0.02$, Figure 4A), despite having similar clinical T stages prior to NA CRT. The number of lymph nodes analysed and lymph node positivity did not differ between normal and overweight/obese cohorts. Overweight/obese patients were more likely to achieve a good TRG response (TRG1-3) compared to normal weight patients ( $R R=2.141, \quad 95 \%$ $\mathrm{CI}=1.374-3.336, p=0.002$, Figure $4 \mathrm{~B})$. When patients with TRG 3 were excluded from the analysis, overweight/obese patients were still more likely to achieve a good (TRG 1-2) response following NA CRT $(\mathrm{RR}=3.259,95 \% \mathrm{CI}=1.269-8.368, p=0.004$, Figure $4 \mathrm{C})$. When classified according to the most recent TRG stratification(29), overweight/obese patients were still more likely to achieve a complete (TRG 1) or partial (TRG 2-3) response following NA CRT $(p=0.0025)$, but the number of patients in each treatment response group was small (Supplementary figure 1). When classified into normal weight, overweight and obese categories, overweight patients were more likely to achieve a good (TRG 1-3) response following NA CRT compared to normal weight patients $(R R=2.147,95 \%$ $\mathrm{CI}=1.294-3.561, p=0.0041$ ) (Supplementary figure 2A). Obese patients were more likely to achieve a good (TRG 1-3) response following NA CRT compared to normal weight patients ( $R R=2.130, \quad 95 \%$ $\mathrm{CI}=1.157-3.923, p=0.01)$, (Supplementary figure 2B). There was no difference in TRG response in overweight compared to obese patients (data not shown).

Direct logistic regression was performed to assess the impact of a number of factors on the likelihood of a good TRG response. The model contained six variables (age, gender, smoking history, ASA grade, clinical T stage, BMI classification). Only BMI classification independently predicted TRG response. Overweight and obese patients were three times more likely to achieve a good TRG response after controlling for all other factors in the model $(\mathrm{OR}=3.376, \quad 95 \% \quad \mathrm{CI}=1.68-6.784, \quad p=0.002)$. Obesity 
status was not associated with risk of recurrence and did not affect survival (Figure 4D). Five-year survival was $21 \%$, with overall median disease-specific survival of 27 months (median survival for normal weight patients was 23 months, overweight/obese median survival was 30 months).

Therefore, clinically we have shown that while there was no difference in preoperative clinical $\mathrm{T}$ stage between normal weight patients and overweight and obese patients, overweight and obese patients were more likely to have a lower pathological $\mathrm{T}$ stage at surgery and to achieve a good TRG response (TRG 1-3) to NA CRT. Obesity status did not influence rates of postoperative morbidity, recurrence or survival.

\section{Discussion}

Adenocarcinoma of the oesopahgus often presents late and confers a poor prognosis. Although response rates to chemotherapy and radiation therapy have improved, treatment resistance is a significant (a)

(c)

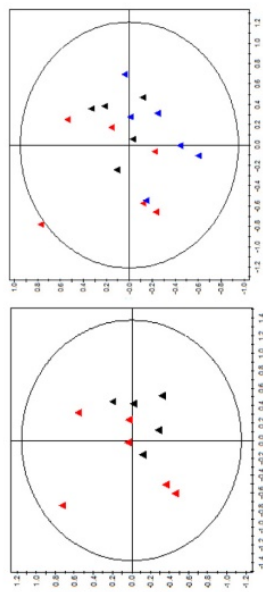

(e)

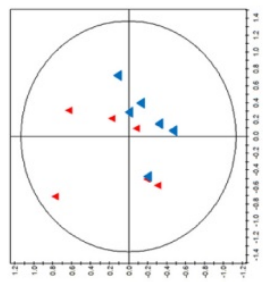

(b)

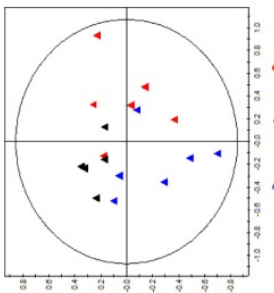

(d)

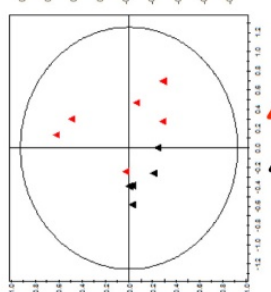

(f)

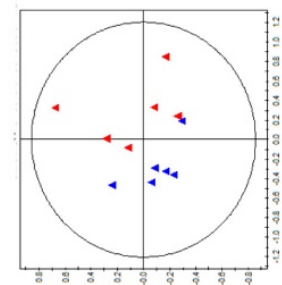

Non cancer patients

Surgery only OAC patients $\triangle$ NA CRT OAC patients

Non cancer patients

Surgery only OAC patients

Non cancer patients $\triangle$ NA CRT OAC patients
Figure 2. The metabolic profile is altered in ACM from non-cancer patients compared to ACM from surgery only OAC patients and NA CRT OAC patients. (A) Principal component analysis (PCA) plot of NMR spectra from non-cancer patients (red triangles, $n=6$ ), surgery only OAC patients (black triangles, $n=6$ ) and NA CRT OAC patients (blue triangles, $n=6)$, showed separation of the three patient cohorts $\left(R^{2}=0.736\right)$. (B) A partial least-squares discriminant analysis (PLS-DA) of IH NMR data from was constructed. Separation of the three cohorts was seen, but the model was weak, $\left(R^{2}=0.453, Q^{2}=0.235\right)$. Therefore, pairwise PCA and PLS-DA plots were constructed. (C) PCA plot of IH NMR ACM data for two patient cohorts (non cancer cohort versus surgery only OAC cohort), $\left(R^{2}=0.699\right)$. (D) PLS-DA of $1 \mathrm{H}$ NMR ACM data for non-cancer cohort versus surgery only $O A C$ cohort, $\left(R^{2}=0.479\right.$, $\left.\mathrm{Q}^{2}=0.304\right)$. (E) PCA plot of non cancer patients versus NA CRT OAC patients $\left(\mathrm{R}^{2}=0.547\right)$. (F) PLS-DA of IH NMR plasma data for non-cancer cohort versus NA CRT OAC cohort, $\left(R^{2}=0.474\right.$, $\mathrm{Q}^{2}=0.318$ ). A representative PCA and PLS-DA model showing separation of the surgery only OAC and NA CRT OAC cohorts was constructed, and separation of the two patient cohorts was seen, but the $\mathrm{Q}^{2}$ value indicated that the model was weak (data not shown). clinical challenge (1). Analysis of standard clinicopathological parameters does not predict response to NA CRT (30). This study demonstrates for the first time a role for obesity in modulating response to radiation. We show that radiosensitivity was in radioresistant OE33R cells treated with radiosensitivity is not merely a bystander effect sed by previous exposure of the fat tissue to ion, but a response induced by factors from the treated with ACM from patients who had received NA CRT, suggesting that previous exposure to ation may have influenced the visceral fat in $\mathrm{ACM}$ from non-cancer patient and surgery only patients also decreased radioresistance in the radioresistant cells, this suggest that factors in the ACM driving this effect are not present as a consequence of malignancy, but may be driven by the inflammatory nature of the visceral fat tissue itself. Factors secreted into the tumour microenvironment such as cyclin D1, GDF-15, VEGF and MMP are known to modulate the radioresponse (34-37). Given the complexity of the cellular response to ionizing radiation, it is likely that multiple interacting processes can regulate sensitivity to radiation.

Radioresistance was significantly lower in OE33R cells treated with ACM from NA CRT patients, compared to ACM from the radiation naïve $\mathrm{OAC}$ cohort, suggesting the ACM from these two cohorts may have distinct metabolic actions: previous radiation may have stimulated the visceral fat to release metabolites which could further alter the radioresponse. Metabolomic profiling did not demonstrate any difference in metabolites between surgery only ACM and NA CRT OAC patient ACM which might account for the difference in radioresponse seen in our isogenic model. However, ACM from surgery only and NA CRT OAC patients was significantly altered compared to non-cancer patients.

Higher levels of threonine, lysine and valine, and lower levels of glucose were seen in the metabolome of both surgery only and NA CRT OAC patients compared to non-cancer patients. A serum metabolite 
signature including elevated levels of threonine and isoleucine predicted response to chemotherapy in breast cancer patients (38). Metabolic profiling of OAC patients demonstrated increased serum levels of lysine and glucose in OAC patients, when compared to healthy controls (39). Previous metabolomic analysis of tumour tissue, serum and urine in oesophageal cancer demonstrated variation in the metabolites of glycolysis, lactic acid fermentation and
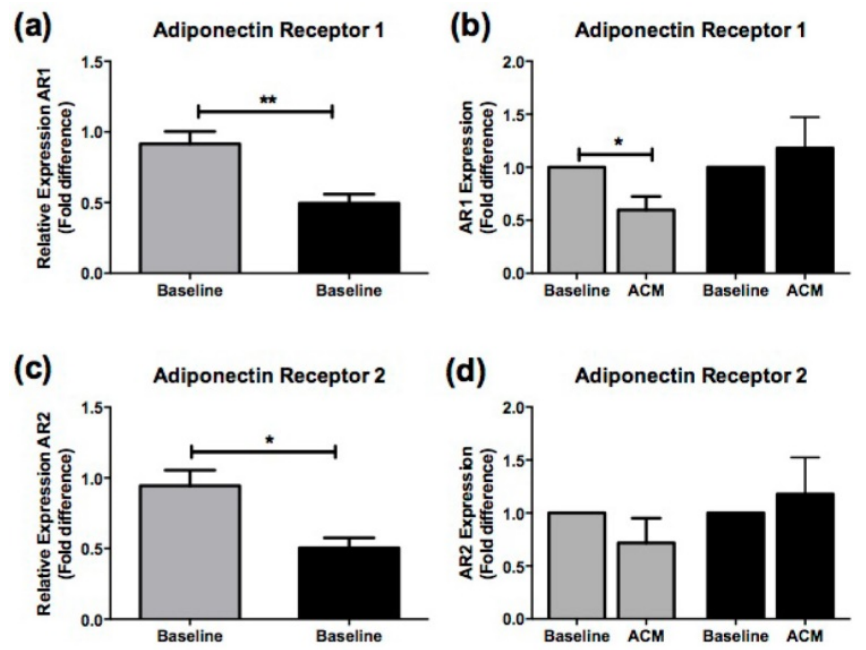

(e)
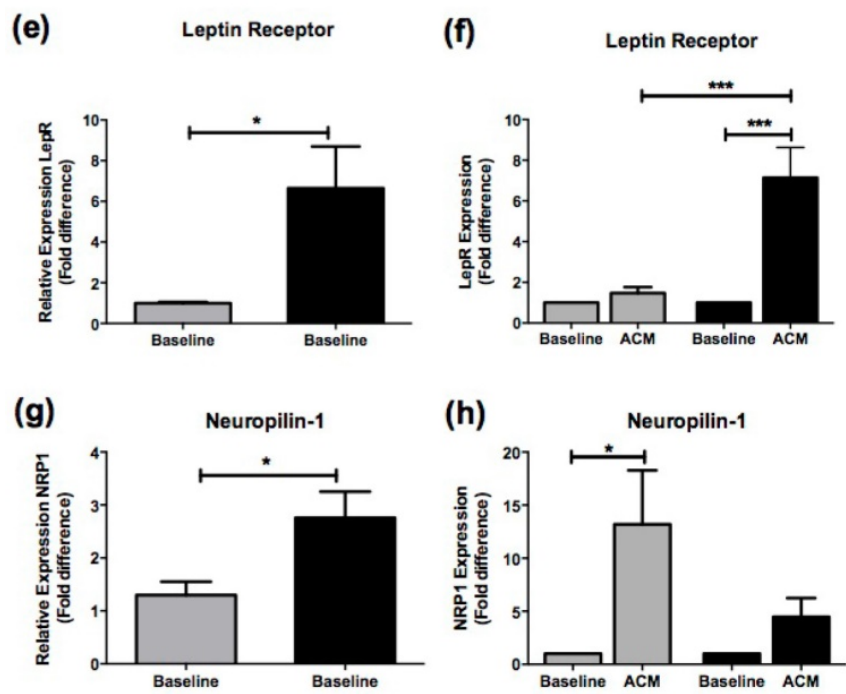

Figure 3. Adipokine receptor expression is altered in radioresistant OAC cells. Expression of a panel of adipokine receptors was assessed in OE33P and OE33R cell lines at baseline and following treatment with ACM from OAC patients, using QPCR. (A) Expression of ARI was significantly higher in OE33P compared to OE33R at baseline $(p=0.008)$. (B) ACM induced a significant downregulation of AR1 expression in OE33P cells $(P=0.019)$ but did not alter AR1 expression in OE33R. (C) Basal AR2 expression is significantly increased in OE33P cells, when compared to OE33R ( $P=0.015$ ). (D) No significant difference in $A R 2$ expression was demonstrated in OE33P or OE33R cells treated with ACM for 24 hours. (E) Basal LepR expression was significantly lower in OE33P, when compared to OE33R $(p=0.033)$. (F) ACM treatment did not alter LepR expression in OE33P. LepR expression was significantly increased in OE33R cells following $A C M$ treatment $(p=0.006)$. LepR expression was significantly increased in OE33R compared to OE33P cells treated with ACM $(p=0.0004)$ (G) NRPI expression was significantly lower in OE33P compared to OE33R $(p=0.038)$. (H) $A C M$ significantly upregulated NRPI expression in OE33P cells $(P=0.047)$, however NRPI expression in OE33R cells was not altered following ACM treatment. Data are expressed as mean \pm SEM. Statistical analysis was performed using unpaired two-tailed student's $t$-test to compare expression in OE33P versus OE $33 \mathrm{R}$ and paired two-tailed student's $t$-test to compare ACM treated cells versus control; ${ }^{p} \mathrm{p}<0.05,{ }^{*} \mathrm{p}<<0.001,{ }^{*} * \mathrm{p} p<0.0001$. amino acid synthesis in oesophago-gastric cancer (40). We have previously shown that altered energy metabolism is associated with radioresistance in OAC (41). Future analysis may allow identification of the metabolic pathways in adipose tissue associated with modulation of cancer risk in obese individuals. Mass spectrometry- based metabolomics has demonstrated utility for identifying biomarkers of ionizing radiation exposure in cellular, mouse and rat in vivo radiation models (42). In addition, metabolomic mapping of ovarian, endometrial, lung, renal and colorectal cancer have demonstrated alterations in serum or urine profiles (43). Systemic alterations in the metabolomic profile of urine and serum may influence the radioresponse in obese individuals; however they cannot explain the local effect of ACM on radioresponse in OAC cells, indicating that other mechanisms must play a greater role, possibly differential expression of adipokine receptors.

Our study demonstrated that baseline adipokine receptor expression differs between OE33P and OE33R. AR1 and AR2 expression was higher in OE33P compared to OE33R, while LepR and NRP1 expression was lower in OE33P compared to OE33R. Furthermore, following ACM treatment, expression of LepR was higher in OE33R compared to OE33P.

Adiponectin is the most abundant adipokine (44), and over $90 \%$ of OACs express AR1 and AR2 (45). High expression of AR1 and AR2 is associated with prolonged survival in lung, thyroid and gastric cancer (46-49). These findings are consistent with our in vitro data demonstrating that AR1 and AR2 levels are higher in radioresponsive compared to radioresistant cancer. Adiponectin has been shown to inhibit tumour growth in animals (44), and recombinant adiponectin has antiproliferative and proapoptotic effects on oesophageal adenocarcinoma cell lines that express both AR1 and AR2 (50,51), which may potentially contribute to delaying recovery from DNA damage following irradiation, enhancing radiosensitivity.

Expression of LepR and NRP1 correlates with aggressive tumour behaviour in $\mathrm{OAC}$, and poor prognosis in a range of cancers $(45,52-59)$, however, their association with radiosensitivity status has not been investigated. We showed that LepR and NRP1 are upregulated in radioresistant 
OAC. We and others have demonstrated that leptin promotes cell proliferation, angiogenesis and metalloproteinase expression in oesophageal and colonic cancer cell lines $(45,60-63)$. The obese $\mathrm{db} / \mathrm{db}$ mouse, which lacks a functional LepR, demonstrates increased susceptibility to radiation (64), and leptin upregulation is known to suppress therapy-induced apoptosis by inhibiting caspase activation (65).

Our in vitro results demonstrating that ACM influences radiosensitivity were further supported by clinical data indicating an association between increased adiposity and improved radioresponse. Only $15-45 \%$ of patients undergoing NA CRT achieve a complete pathologic response and subsequently, have improved outcomes (66). It is not understood why tumours of identical pre-treatment stage, undergoing identical neo-adjuvant regimens, respond differently to NA CRT (66-70). Our study suggests that BMI status influences tumour regression in OAC. We have shown that patients who were overweight or obese were more likely to have a lower pathological $\mathrm{T}$ stage than normal weight patients, despite having similar clinical $\mathrm{T}$ stages prior to treatment, and were more likely to achieve a good tumour regression grade than normal weight patients. Our results were consistent across a number of TRG classification systems. Shridhar et al. specifically examined oncological and survival outcomes in a cohort of OAC
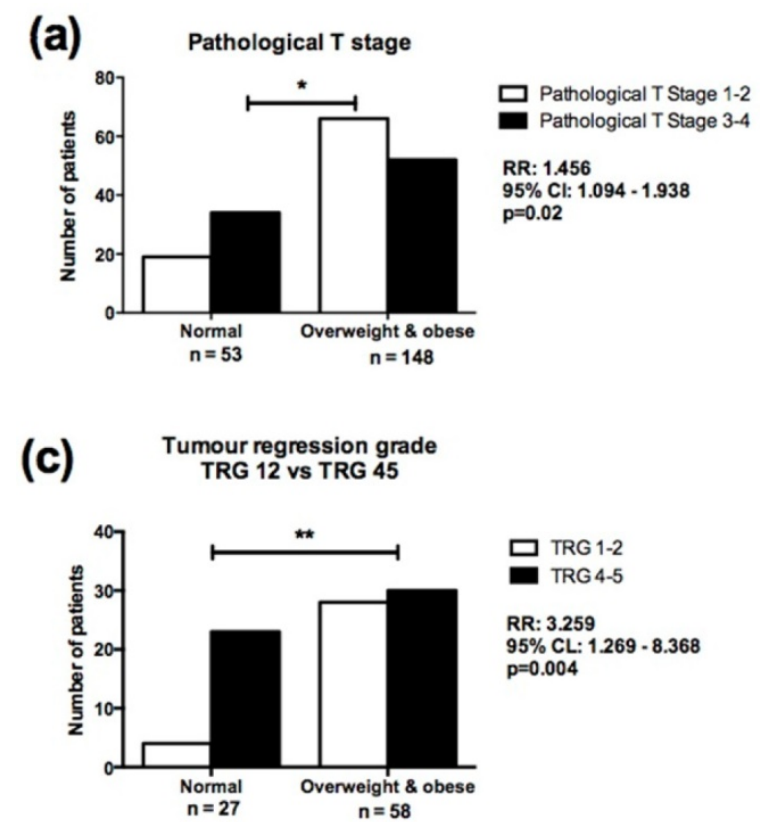

patients who received NA CRT; BMI was not associated with tumour response (13). In our study, obesity status was not associated with recurrence or survival. Four studies investigating outcomes in OAC patients in relation to BMI have included a cohort of patients who received NA CRT, none of which demonstrated a difference in survival between obese and non-obese patients (11-14). Improved TRG outcome indicates improved locoregional controlhowever the effect on survival in obese patients may be offset by associated morbidities, salvage systemic therapies, early metastic events or treatment toxicity. A limitation of this retrospective study is the use of BMI obtained at the time of diagnosis, which might not reflect changes in BMI that occurred before diagnosis, and visceral fat area measurement of obesity might have more closely mirrored the obesity status (71-73) and should be the focus of future studies.

Importantly, this study was strengthened by the focus on a homogeneous patient population of non-metastatic OAC patients that were all treated with NA CRT followed by resection, reducing the possibility of outcome differences due to stage or treatment differences. Our findings linking obesity and radiation response may translate into other cancer types and outcomes.
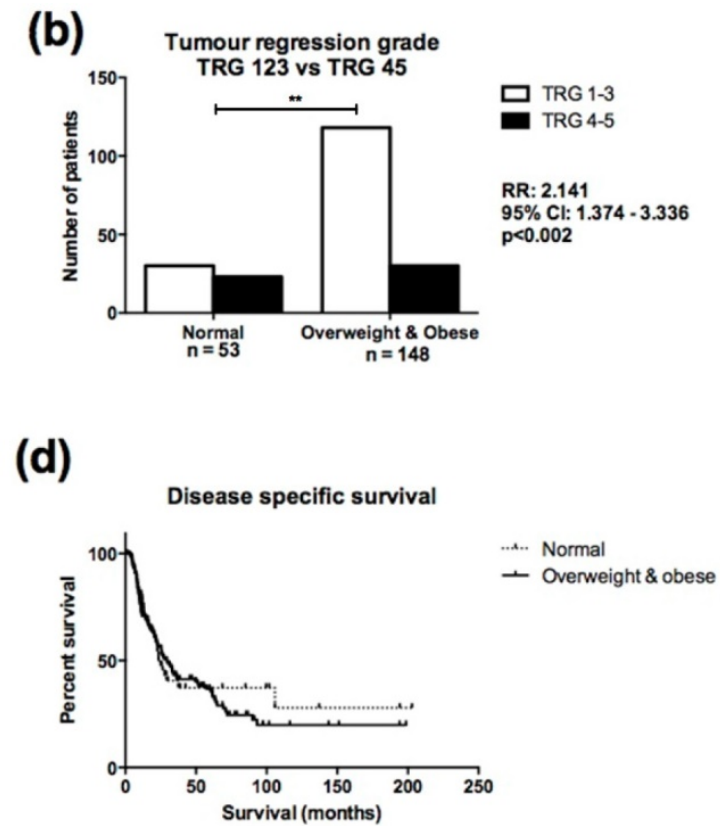

Figure 4. Overweight/obese patients demonstrate enhanced response to NA CRT compared to normal weight OAC patients. (A) Normal weight patients were more likely to have higher pathological T stage (T stage 3-4) at resection, following NA CRT, when compared to overweight/obese patients (RR=1.456, 95\% Cl=1.094-1.938, $\mathrm{p}=0.02$ ). (B) Overweight/obese patients were twice as likely to achieve a good (TRG 1-3) response following NA CRT, when compared to normal weight patients $(R R=2.141,95 \% \mathrm{Cl}=1.374-3.336, p=0.002)$. (C) When patients with TRG 3 were excluded from the analysis, overweight/obese patients were three times as likely to achieve a good (TRG 1-2) response following NA CRT, when compared to normal weight patients $(R R=3.259,95 \% \mathrm{Cl}=1.269-8.368, \mathrm{p}=0.004)$. (D) There was no statistically significant difference in survival in normal weight compared to overweight/obese patients. Median survival was 23 and 30 months respectively. Comparison of patient cohorts in terms of pathological T stage and TRG was performed using $X^{2}$ test. A p value of $\leq 0.05$ was considered to be statistically significant. ${ }^{*} p<0.05, * * p<0.001 ; R R=$ relative risk; $95 \% \mathrm{Cl}=95 \%$ confidence interval. Survival was calculated from date of diagnosis to the first event (i.e. death or end of the follow-up period). Survival analysis was performed using Kaplan-Meier curves and log-rank test. 


\section{Conclusions}

This study suggests a link between obesity status and response to radiation. Further work is warranted to determine functionally the connection between these two processes.

\section{Supplementary Material}

Supplementary figures and tables. http://www.medsci.org/v16p0519s1.pdf

\section{Acknowledgements}

We would like to thank the members of the oesophageal surgical team at St. James's Hospital. We would also like to thank all the patients who gave their consent for sample procurement. This work was supported by funding from the Health Research Board (HPF / 2011/59).

\section{Competing Interests}

The authors have declared that no competing interest exists.

\section{References}

1. Enzinger PC, Mayer RJ. Esophageal cancer. N Engl J Med. 2003 Dec;349(23):2241-52

2. van Hagen $P$, Hulshof $M C$, van Lanschot JJ et al. Preoperative chemoradiotherapy for esophageal or junctional cancer. N Engl J Med. 2012 May 31;366(22):2074-84.

3. Oppedijk V, van der Gaast A, van Lanschot JJB, et al. Patterns of recurrence after surgery alone versus preoperative chemoradiotherapy and surgery in the CROSS trials. J Clin Oncol. 2014 Feb;32(5):385-91.

4. Sjoquist KM, Burmeister BH, Smithers BM et al. Survival after neoadjuvant chemotherapy or chemoradiotherapy for resectable oesophageal carcinoma: an updated meta-analysis. Lancet Oncol. 2011 Jul;12(7):681-92.

5. Renehan AG, Tyson M, Egger M et al. Body-mass index and incidence of cancer: a systematic review and meta-analysis of prospective observational studies. Lancet. 2008 Feb 16;371(9612):569-78.

6. Calle EE, Kaaks R. Overweight, obesity and cancer: epidemiological evidence and proposed mechanisms. Nat Rev Cancer. 2004 Aug:4(8):579-91.

7. Lagergren J. Adenocarcinoma of oesophagus: what exactly is the size of the problem and who is at risk? Gut. 2005 Mar;54 (Suppl 1):i1-5.

8. Sinicrope F, Foster NR, Sargent DJ et al. Obesity is an independent prognostic variable in colon cancer surviviors. Clin Cancer Res. 2010 Mar 15;16(6):1884-93

9. Palma D, Pickles T \& Tyldesley S; Prostate Cohort Outcomes Initiative. Obesity as a predictor of biochemical recurrence and survival after radiation therapy for prostate cancer. BJU Int. 2007 Aug;100(2):315-9

10. Meyerhardt JA, Tepper JE, Niedwiecki D et al. Impact of body mass index on outcomes and treatment-relatedtoxicity in patients with stage II and III rectal cancer: findings from Intergroup Trial 0114. J Clin Oncol. 2004 Feb 15;22(4):648-57

11. Healy LA, Ryan AM, Gopinath B et al. Impact of obesity on outcomes in the management of localized adenocarcinoma of the esophagus and esophagogastric junction. J Thorac Cardiovasc Surg. 2007 Nov;134(5):1284-1291

12. Morgan MA, Lewis WG, Hopper AN et al. Prognostic significance of body mass indices for patients undergoing esophagectomy for cancer. Dis Esophagus. 2007;20(1):29-35

13. Shridhar R, Hayman T, Hoffe SE et al. Body mass index and survival in esophageal adenocarcinoma treated with chemoradiotherapy followed by esophagectomy. J Gastrointest Surg. 2012 Jul;16(7):1296-302

14. Wang J, Myles B, Wei C et al. Obesity and outcomes in patients treated with chemoradiotherapy for esophageal carcinoma. Dis Esophagus. 2014;27(2):168-75

15. Maity A, Kao GD, Muschel RJ et al. Potential molecular targets for manipulating the radiation response. Int J Radiat Oncol Biol Phys. 1997 Feb;37(3):639-53.

16. Mcilrath J, Bouffler SD, Samper E et al. Telomere Length Abnormalities in Mammalian Radiosensitive Cells. Cancer Res. 2001;61(3):912-5.

17. Diehn M, Cho RW, Lobo NA et al. Association of reactive oxygen species levels and radioresistance in cancer stem cells. Nature. 2009 Apr 9;458(7239):780-3.
18. Furukawa $\mathrm{S}$, Fujita $\mathrm{T}$, Shimabukuro $\mathrm{M}$ et al. Increased oxidative stress in obesity and its impact on metabolic syndrome. J Clin Invest. 2004 Dec;114(12):1752-61.

19. Fyhrquist F, Saijonmaa O. Telomere length and cardiovascular aging. Ann Med. 2012;44:S138-42.

20. McCullough LE, Eng SM, Bradshaw PT et al. Genetic polymorphisms in DNA repair and oxidative stress pathways may modify the association between body size and postmenopausal breast cancer. Ann Epidemiol. 2015 Apr;25(4):263-9.

21. Lynam-Lennon N, Reynolds J V, Pidgeon GP et al. Alterations in DNA repair efficiency are involved in the radioresistance of esophageal adenocarcinoma. Radiat Res. 2010 Dec;174(6):703-11.

22. Donohoe CL, Doyle SL, Reynolds J V. Visceral adiposity, insulin resistance and cancer risk. Diabetol Metab Syndr. 2011 Jan;3(1):12.

23. Allott EH, Morine MJ, Lysaght J et al. Elevated Tumor Expression of PAI-1 and SNAI2 in Obese Esophageal Adenocarcinoma Patients and Impact on Prognosis. Clin Transl Gastroenterol. 2012 Jan;3:e12.

24. Lysaght J, van der Stok EP, Allott EH et al. Pro-inflammatory and tumour proliferative properties of excess visceral adipose tissue. Cancer Lett. 2011 Dec 15;312(1):62-72.

25. Lynam-Lennon $\mathrm{N}$, Connaughton $\mathrm{R}$, Carr $\mathrm{E}$ et al. Excess visceral adiposity induces alterations in mitochondrial function and energy metabolism in esophageal adenocarcinoma. BMC Cancer. 2014 Jan;14:907.

26. Doyle SL, Bennett AM, Donohoe CL et al. Establishing computed tomography-defined visceral fat area thresholds for use in obesity-related cancer research. Nutr Res. 2013 Mar;33(3):171-9.

27. Miller KD, Jones E, Yanovski JA et al. Visceral abdominal-fat accumulation associated with use of indinavir. Lancet. 1998 Mar 21;351(9106):871-5.

28. Baumgartner RN, Heymsfield SB, Roche AF et al. Abdominal composition quantified by computed tomography. Am J Clin Nutr . 1988 Oct;48(4):936-45.

29. Ennis DP, Pidgeon GP, Millar N et al. Building a bioresource for esophageal research: lessons from the early experience of an academic medical center. Dis Esophagus. 2010 Jan;23(1):1-7.

30. Sobin LH, Gospodarowicz M, Wittekind C, editors. Wiley: TNM Classification of Malignant Tumours, 7th Edition - Leslie H. Sobin, Mary K. Gospodarowicz, Christian Wittekind. 7th Edition. Oxford; 2009

31. Mandard AM, Dalibard F, Mandard JC, Marnay J, Henry-Amar M, Petiot JF, et al. Pathologic assessment of tumor regression after preoperative chemoradiotherapy of esophageal carcinoma. Clinicopathologic correlations. Cancer. 1994 Jun 1;73(11):2680-6.

32. Donohoe $\mathrm{CL}, \mathrm{O}^{\prime}$ Farrell NJ, Grant $\mathrm{T}$ et al. Classification of pathologic response to neoadjuvant therapy in esophageal and junctional cancer: assessment of existing measures and proposal of a novel 3-point standard. Ann Surg. 2013 Nov;258(5):784-92

33. Chirieac LR, Swisher SG, Ajani JA et al. Posttherapy pathologic stage predicts survival in patients with esophageal carcinoma receiving preoperative chemoradiation. Cancer. 2005 Apr 1;103(7):1347-55.

34. Zhang L, Ge $\mathrm{W}$, $\mathrm{Hu} \mathrm{K}$ et al. Endostar down-regulates HIF-1 and VEGF expression and enhances the radioresponse to human lung adenocarcinoma cancer cells. Mol Biol Rep. 2012;39(1):89-95

35. Hinz M, Krappmann D, Eichten A et al. NF-kappaB function in growth control: regulation of cyclin D1 expression and G0/G1-to-S-phase transition. Mol Cell Biol. 1999 Apr;19(4):2690-8.

36. Wen QL, Meng MB, Yang B et al. Endostar, a recombined humanized endostatin, enhances the radioresponse for human nasopharyngeal carcinoma and human lung adenocarcinoma xenografts in mice. Cancer Sci. 2009 Aug;100(8):1510-9

37. Sándor N, Schilling-Tóth B, Kis E et al. Growth Differentiation Factor-15 (GDF-15) is a potential marker of radiation response and radiation sensitivity. Mutat Res Genet Toxicol Environ Mutagen. 2015 Nov;793:142-9.

38. Wei S, Liu L, Zhang J et al. Metabolomics approach for predicting response to neoadjuvant chemotherapy for breast cancer. Mol Oncol. 2013 Jun 6;7(3):297-307.

39. Zhang J, Liu L, Wei S et al. Metabolomics study of esophageal adenocarcinoma. J Thorac Cardiovasc Surg. 2011 Feb;141(2):469-75, 475-4.

40. Abbassi-Ghadi N, Kumar S, Huang J et al. Metabolomic profiling of oesophago-gastric cancer: a systematic review. Eur J Cancer. 2013;49(17):3625-37.

41. Lynam-Lennon N, Maher SG, Maguire A et al. Altered mitochondrial function and energy metabolism is associated with a radioresistant phenotype in oesophageal adenocarcinoma. PLoS One. 2014 Jan;9(6):e100738.

42. Johnson $\mathrm{CH}$, Patterson $\mathrm{AD}$, Krausz KW et al. Radiation metabolomics. 5. Identification of urinary biomarkers of ionizing radiation exposure in nonhuman primates by mass spectrometry-based metabolomics. Radiat Res. 2012 Oct; $178(4): 328-40$

43. Nicholson JK, Holmes E, Kinross JM et al. Metabolic phenotyping in clinical and surgical environments. Nature. 2012 Nov 15;491(7424):384-92.

44. Rose DP, Komninou D, Stephenson GD. Obesity, adipocytokines, and insulin resistance in breast cancer. Obes Rev. 2004 Aug:5(3):153-65.

45. Howard JM, Beddy P, Ennis D et al. Associations between leptin and adiponectin receptor upregulation, visceral obesity and tumour stage in oesophageal and junctional adenocarcinoma. Br J Surg. 2010 Jul;97(7):1020-7.

46. Abdul-Ghafar J, Park S, Wairagu P. Expression of Adiponectin Receptor 1 Is Indicative of Favorable Prognosis in Non-small Cell Lung Carcinoma. Tohoku J Exp Med. 2012;153-62. 
47. Barresi V, Grosso M, Giuffrè G et al. The expression of adiponectin receptors Adipo-R1 and Adipo-R2 is associated with an intestinal histotype and longer survival in gastric carcinoma. J Clin Pathol. 2009 Aug;62(8):705-9.

48. Tsukada T, Fushida S, Harada $\mathrm{S}$ et al. Adiponectin receptor-1 expression is associated with good prognosis in gastric cancer. J Exp Clin Cancer Res. 2011 Jan;30:107.

49. Cheng S-P, Liu C-L, Hsu Y-C et al. Expression and biologic significance of adiponectin receptors in papillary thyroid carcinoma. Cell Biochem Biophys. 2013 Mar;65(2):203-10.

50. Konturek PC, Burnat G, Rau T et al. Effect of adiponectin and ghrelin on apoptosis of Barrett adenocarcinoma cell line. Dig Dis Sci. 2008 Mar;53(3):597-605.

51. Ogunwobi OO, Beales ILP. Globular adiponectin, acting via adiponectin receptor-1, inhibits leptin-stimulated oesophageal adenocarcinoma cell proliferation. Mol Cell Endocrinol . 2008 Mar 26;285(1-2):43-50.

52. Escudero-Esparza A, Martin TA, Douglas-Jones A et al. PGF isoforms, PLGF-1 and PGF-2 and the PGF receptor, neuropilin, in human breast cancer: prognostic significance. Oncol Rep. 2010 Feb;23(2):537-44.

53. Ghosh S, Sullivan CAW, Zerkowski MP et al. High levels of vascular endothelial growth factor and its receptors (VEGFR-1, VEGFR-2, neuropilin-1) are associated with worse outcome in breast cancer. Hum Pathol. 2008 Dec;39(12):1835-43.

54. Broholm H, Laursen H. Vascular endothelial growth factor (VEGF) receptor neuropilin-1's distribution in astrocytic tumors. APMIS. 2004;112(4-5):257-63.

55. Stephenson JM, Banerjee S, Saxena NK et al. Neuropilin-1 is differentially expressed in myoepithelial cells and vascular smooth muscle cells in preneoplastic and neoplastic human breast: a possible marker for the progression of breast cancer. Int J Cancer. 2002 Oct 10;101(5):409-14.

56. Hansel DE, Wilentz RE, Yeo CJ et al. Expression of neuropilin-1 in high-grade dysplasia, invasive cancer, and metastases of the human gastrointestinal tract. Am J Surg Pathol. 2004 Mar;28(3):347-56.

57. Lantuéjoul S, Constantin B, Drabkin H et al. Expression of VEGF, semaphorin SEMA3F, and their common receptors neuropilins NP1 and NP2 in preinvasive bronchial lesions, lung tumours, and cell lines. J Pathol. 2003 Jul;200(3):336-47.

58. Latil A, Bièche I, Pesche $\mathrm{S}$ et al. VEGF overexpression in clinically localized prostate tumors and neuropilin-1 overexpression in metastatic forms. Int J Cancer. 2000 Mar 20;89(2):167-71.

59. Parikh AA, Fan F, Liu WB et al. Neuropilin-1 in human colon cancer: expression, regulation, and role in induction of angiogenesis. Am J Pathol. 2004 Jun;164(6):2139-51.

60. Somasundar P, McFadden DW, Hileman SM et al. Leptin is a growth factor in cancer. J Surg Res. 2004 Mar;116(2):337-49.

61. Park HY, Kwon HM, Lim HJ et al. Potential role of leptin in angiogenesis: leptin induces endothelial cell proliferation and expression of matrix metalloproteinases in vivo and in vitro. Exp Mol Med. 2001 Jun 30;33(2):95-102.

62. Ogunwobi OO, Beales ILP. Leptin stimulates the proliferation of human oesophageal adenocarcinoma cells via HB-EGF and Tgfalpha mediated transactivation of the epidermal growth factor receptor. Br J Biomed Sci. 2008 Jan;65(3):121-7.

63. Healy LA, Howard JM, Ryan AM et al. Metabolic syndrome and leptin are associated with adverse pathological features in male colorectal cancer patients. Colorectal Dis. 2012 Feb;14(2):157-65.

64. Gove ME, Sherry CL, Pini M et al. Generation of leptin receptor bone marrow chimeras: recovery from irradiation, immune cellularity, cytokine expression, and metabolic parameters. Obesity (Silver Spring) . 2010 Dec;18(12):2274-81.

65. Jiang $\mathrm{H}, \mathrm{Yu} \mathrm{J}$, Guo $\mathrm{H}$ et al. Upregulation of survivin by leptin/STAT3 signaling in MCF-7 cells. Biochem Biophys Res Commun. 2008 Mar 28;368(1):1-5.

66. Gebski V, Burmeister B, Smithers BM et al. Survival benefits from neoadjuvant chemoradiotherapy or chemotherapy in oesophageal carcinoma: a meta-analysis. Lancet Oncol. 2007 Mar;8(3):226-34.

67. Geh JI, Bond SJ, Bentzen SM et al. Systematic overview of preoperative (neoadjuvant) chemoradiotherapy trials in oesophageal cancer: evidence of a radiation and chemotherapy dose response. Radiother Oncol. 2006 Mar;78(3):236-44.

68. Cheedella NKS, Suzuki A, Xiao L et al. Association between clinical complete response and pathological complete response after preoperative chemoradiation in patients with gastroesophageal cancer: analysis in a large cohort. Ann Oncol. 2013 May;24(5):1262-6.

69. Walsh TN, Noonan N, Hollywood D et al. A comparison of multimodal therapy and surgery for esophageal adenocarcinoma. N Engl J Med. 1996 Aug 15;335(7):462-7.

70. Luu TD, Gaur P, Force SD et al. Neoadjuvant chemoradiation versus chemotherapy for patients undergoing esophagectomy for esophageal cancer. Ann Thorac Surg. 2008 Apr;85(4):1217-23-4.

71. Balentine CJ, Marshall C, Robinson C, Wilks J, Anaya D, Albo D, et al. Validating quantitative obesity measurements in colorectal cancer patients. J Surg Res. 2010 Nov;164(1):18-22.

72. Rössner S, Bo WJ, Hiltbrandt E, Hinson W, Karstaedt N, Santago P, et al. Adipose tissue determinations in cadavers--a comparison between cross-sectional planimetry and computed tomography. Int J Obes. 1990 Oct;14(10):893-902.
73. Klopfenstein BJ, Kim MS, Krisky CM, Szumowski J, Rooney WD, Purnell JQ. Comparison of $3 \mathrm{~T}$ MRI and CT for the measurement of visceral and subcutaneous adipose tissue in humans. $\mathrm{Br} J$ Radiol. 2012 Oct; $85(1018): e 826-30$ 